\title{
Gastrointestinal Stromal Tumor Mimicking Ovarian Tumor
}

\author{
Priyanka Kadam*, Pariseema S. Dave, Meeta H. Mankad \\ Department of Gynaecologic Oncology, Gujarat Cancer and Research Institute, Ahmedabad, India \\ Email: ${ }^{*}$ priyankakadam575@gmail.com
}

Received 29 July 2015; accepted 13 September 2015; published 16 September 2015

Copyright (C) 2015 by authors and Scientific Research Publishing Inc.

This work is licensed under the Creative Commons Attribution International License (CC BY). http://creativecommons.org/licenses/by/4.0/

(c) $\underset{\mathrm{EY}}{\mathrm{E}}$ Open Access

\begin{abstract}
Background: Gastrointestinal stromal tumors (GIST) are rare mesenchymal tumors that may mimic ovarian tumor on presurgical testing. They are most often located in the stomach and proximal small intestine. The gross appearance of GISTs is highly variable. These tumors are usually asymptomatic, often discovered accidentally during endoscopic or radiologic examinations. Case Report: We reported a case of 55-year-old post menopausal woman who presented to us as ovarian mass which turned out to be malignant epithelioid gastrointestinal stromal tumor intraoperatively and by immunohistochemistry. Conclusion: In the patients with a pelvic mass, especially if unusual symptoms and laboratory data being not compatible with gynecological disease, the possibility of diseases other than a gynecologic disease has to be considered. Considering that only few patients with gastrointestinal stromal tumors have been reported in the obstetrical and gynecological literature, the awareness of such an entity by the obstetricians-gynecologists is necessary in order to facilitate coordinated approach with the general surgeons and oncologists for the optimal care of the patients.
\end{abstract}

\section{Keywords}

\section{Gastrointestinal Stromal Tumor}

\section{Introduction}

GISTs (Gastrointestinal stromal tumors) are most common mesenchymal neoplasms of gastrointestinal tract. They accounts for $<1 \%$ of all Gastrointestinal (GI) tumors and its incidence is approximately 10 - 20 per million people per year. These are believed to originate from the interstitial cells of Cajal (ICCs) or from the precursors of ICCs [1]. Most GISTs show an activating mutation in either the c-kit or platelet-derived growth factor receptor alpha (PDGFRA) gene [2]. Morphologically, GISTs vary from cellular spindle cell tumors (70\% - 80\%) to

*Corresponding author. 
epithelioid (20\% - 30\%) or mixed. Gastrointestinal stromal tumours (GISTs) were initially presumed to be of "true" smooth muscle origin, so they were classified as leiomyomas, leiomyosarcomas and leiomyoblastomas [3]. Loss of succinate dehydrogenase complex is a newly understood pathogenesis for $5 \%$ of GIST, associated with early onset of disease [4]. These are mostly asymptomatic and found incidentally during laparoscopy, surgical procedures or radiological studies. Diagnosis is based on histology and immunohistochemistry, while the role of imaging studies is not specific. Ultrasound and MRI are not able to differentiate a GIST from ovarian cancer. Surgical resection of the local disease is the mainstay therapy. However, therapeutic agents such as Imatinib have now been approved for the treatment of advanced GISTs. These tumors occasionally present a pelvic mass and lead to the misdiagnosis of gynecologic diseases. Our case was also presented as ovarian mass which turned out to be malignant epithelioid gastrointestinal stromal tumor intraoperatively and by immunohistochemistry.

\section{Case Report}

A 55-year-old multiparous postmenopausal female presented to us with history of lower abdominal pain, decrease in appetite, constipation and weight loss since 2 months. On clinical examination her vitals were normal and a soft large mobile mass was felt arising from the pelvis. Her CA-125 (20 U/ml) and CA 19-9 (0.6 U/ml) were normal but CEA was raised $(12.99 \mathrm{ng} / \mathrm{ml})$. MRI pelvis revealed altered signal intensity mass of size $15 \times$ $11 \mathrm{~cm}$ in axial plane and $16 \times 10 \mathrm{~cm}$ in sagittal plane extending upto midabdomen arising from pelvis, suggesting possibility of ovarian mass . She was taken for exploratory laparotomy with provisional diagnosis of malignant ovarian tumor. On exploration uterus and both ovaries were found to be normal but a large retroperitoneal mass of size $28 \mathrm{~cm} \times 22 \mathrm{~cm}$ which was adherent to small bowel was seen. Resection of retroperitoneal mass was done which was followed by total abdominal hysterectomy with bilateral salphingo-oophorectomy and infracolic omentectomy. While resecting the retroperitoneal mass, a small rent was present over the small intestine which was repaired. Histopathology report showed the retroperitoneal mass to be a malignant epithelioid gastrointestinal tumor. Her cytology report was negative. Histologically the tumor showed the mass to be a malignant epitheioid gastrointestinal stromal tumor with mitoses in 8 - 9 of 10 high-power fields (Figure 1). Immunohistochemical study showed positivity for c-kit (CD117) and S-100 with neural and muscle differentiation present. A complete cyto immunohistochemical evaluation is mandatory to make an accurate diagnosis. Her post operative period was uneventful.

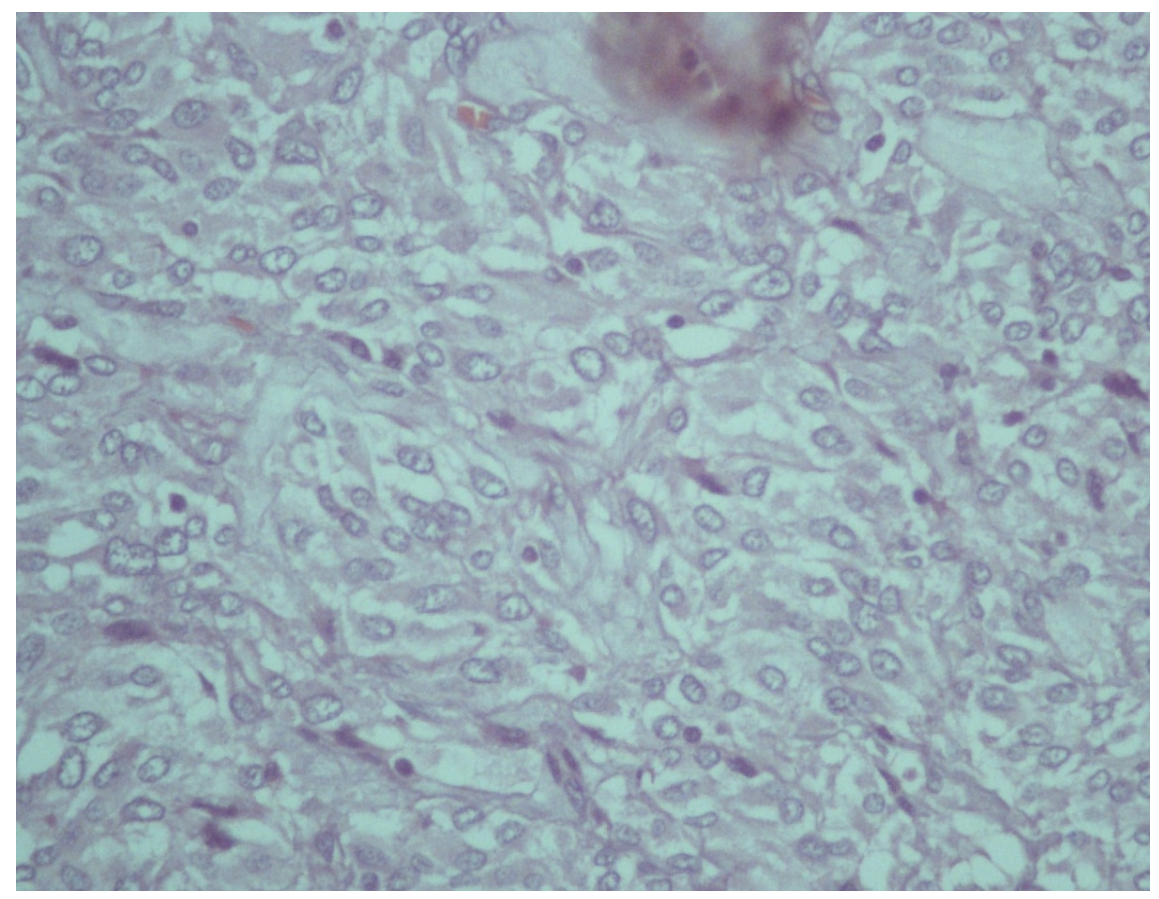

Figure 1. Histology showing 8 - 9 mitosis/10 hpf. 


\section{Discussion}

The malignant epitheloid gastrointestinal stromal tumor is regarded as a rare malignancy that should be distinguished from leiomyomas and leiomyosarcomas as well as from neurinomas. GISTs typically present in adults over 40 years (median age 55 - 60 years) and only exceptionally in children [5]. The clinical signs and symptoms are related to the presence of a mass or bleeding [6]. Studies showed $10 \%$ cases remain asymptomatic because of their small size $(<2 \mathrm{~cm})$ and are diagnosed incidentally [7]. Bleeding comprises the most common symptom and it is attributed to the erosion of the gastrointestinal tract lumen causing haematesis, malena or anaemia. Another common finding is the abdominal mass. However, most of the patients present with vague symptoms, such as nausea, vomiting, abdominal discomfort, weight loss or early satiety. These tumors occasionally present a pelvic mass and leading to the misdiagnosis of gynecologic diseases. The age and clinical presentation of our case was consistent with these reports which mimic the symptoms of ovarian mass. Rupture of GISTs into the peritoneal cavity is rare and it causes life threatening intraperitoneal hemorrhage [8]. A great majority of GISTs occur in the stomach (60\% - 70\%) and small intestine (25\% - 35\%). Colon, rectum, appendix (together 5\%) and esophagus (2\% - 3\%) are the rare sites [8].

In cases in which histological findings are not clear, the unambiguous evidence is provided by immunohistochemical methods, e.g., detection of CD 34 or CD 117 antigens. GISTs are positive for CD117 (95\%), nestin (90\% - 100\%), CD34 (70\%), smooth muscle actins (20\% - 30\%) and heavy caldesmon (80\%) whereas desmin is usually absent. Lymph nodes metastases are not common in GISTs. On the other hand, distant metastases most commonly occur in GIST tumors of peritoneum, omentum, mesenteric areas and liver. Generally, GIST vary greatly in size from few millimeters to $>30 \mathrm{~cm}$ with median size between $5 \mathrm{~cm}$ and $8 \mathrm{~cm}$. Thus, there is not an accepted staging system for GIST. Our case also shown positivity for CD 117 with infiltration to omentum and small bowel no lymph node involvement and .Multiple parameters have been considered as predictors of malignancy. At present, size and mitotic count appear to be the most useful predictors of the malignant behavior [9]. GISTs always have a malignant potential, even if they appear benign. Tumor is considered as benign $(<5 \mathrm{~cm}$ in size and $<5$ mitosis/50 HPF), malignant ( $>5$ mitosis/50 HPF), and borderline lesions ( $>5 \mathrm{~cm}$ and $<5$ mitosis/50 HPF) [10]. Even though size $<5 \mathrm{~cm}$ is reassuring, we cannot always predict them as benign, as there is always the chance to metastasize [11].

Although, the diagnostic procedure includes several examinations like barium examination of the GI tract, computer tomography and angiograph but none of them can establish the diagnosis. Like in our case both ultrasonography and MRI were not able to confirm the diagnosis of GIST and showed possibility of ovarian mass. The preoperative percutaneous biopsy should not be used because it is associated with a significant risk of tumor rupture or dissemination. The significance of endoscopic ultra-sound guided fine needle aspiration has been pointed out in several studies and the reported accuracy is $80 \%$ - 85\% [12]. Endoscopic examinations and fused PET/CT imaging are the most useful techniques for imaging and monitoring the disease progression.

Standard treatment for primary gastrointestinal stromal tumor (GIST) is complete surgical resection, with the aim to obtain negative microscopic margins over the organ of origin. Imatinib mesylate is a very active agent for tumor control in advanced and metastatic GIST [13]. GISTs have a high risk of metastatic relapse. The usual site of recurrence is the liver (65\%), the peritoneal surface (50\%) and both (20\%). The 2-year survival after Imatinib therapy is approximately $70 \%$ and $50 \%$ of the patients showed no progression of the disease [14]. Imatinib interruption after 1 year is associated with a high risk of relapse, even for patients in complete remission [15]. This patient was started Imatinib chemotherapy but after showing the resistance to this therapy patient was put on Dactinomycin. Patient is on regular follow up and is well.

\section{Conclusion}

In the patients with a pelvic mass, especially if unusual symptoms and laboratory data being not compatible with gynecological disease, the possibility of diseases other than a gynecologic disease has to be considered. It should be noted that a preoperative diagnosis of GISTs is uncommon, due to the rarity and many presentations of the disease. Ultrasound and MRI are not able to differentiate a GIST from ovarian cancer. However, if a pelvic mass is detected, the possibility of a non-gynaecological tumor has to be considered.

\section{References}

[1] Yantiss, R.K., Rosenberg, A.E., Selig, M.K. and Nielsen, G.P. (2002) Gastrointestinal Stromal Tumors: An Ultrastruc- 
tural Study. International Journal of Surgical Pathology, 10, 101-113. http://dx.doi.org/10.1177/106689690201000203

[2] Agaram, N.P., Besmer, P., Wong, C.C., Guo, T.H., Socci, N.D., et al. (2007) Pathologic and Molecular Heterogeneity in Imatinib-Stable or Imatinib-Responsive Gastrointestinal Stromal Tumors. Clinical Cancer Research, 13, 170-181. http://dx.doi.org/10.1158/1078-0432.CCR-06-1508

[3] Graadt van Roggen, J.F., van Velthuysen, M.L. and Hogendoorn, P.C. (2001) The Histopathological Differential Diagnosis of Gastrointestinal Stromal Tumours. Journal of Clinical Pathology, 54, 96-102. http://dx.doi.org/10.1136/jcp.54.2.96

[4] Miettinen, M. (2012) [Gastrointestinal stromal tumor]. Duodecim, 128, 1441-1450.

[5] Miettinen, M. and Lasota, J. (2003) Gastrointestinal Stromal Tumors (GISTs): Definition, Occurrence, Pathology, Differential Diagnosis and Molecular Genetics. Polish Journal of Pathology, 54, 3-24.

[6] Heinrich, M.C., Corless, C.L., Duensing, A., McGreevey, L., Chen, C.J., Joseph, N., Singer, S., Griffith, D.J., Haley, A., Town, A., Demetri, G.D., Fletcher, C.D. and Fletcher, J.A. (2003) PDGFRA Activating Mutations in Gastrointestinal Stromal Tumors. Science, 299, 708-710. http://dx.doi.org/10.1126/science.1079666

[7] Kim, K.M., Kang, D.W., Moon, W.S., Park, J.B., Park, C.K., Sohn, J.H., Jeong, J.S., Cho, M.Y., Jin, S.Y., Choi, J.S. and Kang, D.Y. (2005) Gastrointestinal Stromal Tumor Committee; The Korean Gastrointestinal Pathology Study Group. Gastrointestinal Stromal Tumors in Koreans: Incidence and the Clinical, Pathologic and Immunohistochemical Findings. Journal of Korean Medical Science, 20, 977-984. http://dx.doi.org/10.3346/jkms.2005.20.6.977

[8] Motegi, A., Sakurai, S., Nakayama, H., Sano, T., Oyama, T. and Nakajima, T. (2005) PKC Theta, a Novel Immunohistochemical Marker for Gastrointestinal Stromal Tumors (GIST), Especially Useful for Identifying KIT-Negative Tumors. Pathology International, 55, 106-112. http://dx.doi.org/10.1111/j.1440-1827.2005.01806.x

[9] Gupta, P., Tewari, M. and Shulka, H. (2008) Gastrointestinal Stromal Tumor. Surgical Oncology, 17, 129-138. http://dx.doi.org/10.1016/j.suronc.2007.12.002

[10] Ma, C.K., Amin, M.B., Kintanar, E., Linden, M.D. and Zarbo, R.J. (1993) Immunohistologic Characterization of Gastrointestinal Stromal Tumors: A Study of 82 Cases Compared with 11 Cases of Leiomyomas. Modern Pathology, 6, 139-144.

[11] Van Oosterom, A.T., Judson, I., Verweij, J., Stroobants, S., Donato di Paola, E., Dimitrijevic, S., Martens, M., Webb, A., Sciot, R., Van Glabbeke, M., Silberman, S. and Nielsen, O.S. (2001) European Organisation for Research and Treatment of Cancer Soft Tissue and Bone Sarcoma Group. Safety and Efficacy of Imatinib (STI571) in Metastatic Gastrointestinal Stromal Tumours: A Phase I Study. The Lancet, 358, 1421-1423.

[12] Blay, J.Y., Bonvalot, S., Casali, P., Choi, H., Debiec-Richter, M., DeiTos, A.P., et al. (2005) GIST Consensus Meeting Panelists. Consensus Meeting for the Management of Gastrointestinal Stromal Tumors. Report of the GIST Consensus Conference of 20-21 March 2004, under the Auspices of ESMO. Annals of Oncology, 16, 566-578. http://dx.doi.org/10.1093/annonc/mdi127

[13] Verweij, J., Casali, P.G., Zalcberg, J., LeCesne, A., Reichardt, P., et al. (2004) Progression-Free Survival in Gastrointestinal Stromal Tumours with High-Dose Imatinib: Randomised Trial. The Lancet, 364, 1127-1134. http://dx.doi.org/10.1016/S0140-6736(04)17098-0

[14] Joensuu, H. (2006) Gastrointestinal Stromal Tumor (GIST) Gastrointestinal Stromal Tumor (GIST). Annals of Oncology, 17, 280-286. http://dx.doi.org/10.1093/annonc/mdl274

[15] Blay, J.Y., Le Cesne, A., Ray-Coquard, I., Bui, B., Duffaud, F., Delbaldo, C., et al. (2007) Prospective Multicentric Randomized Phase III Study of Imatinib in Patients with Advanced Gastrointestinal Stromal Tumors Comparing Interruption versus Continuation of Treatment beyond 1 Year: The French Sarcoma Group. Journal of Clinical Oncology, 25, 1107-1113. http://dx.doi.org/10.1200/JCO.2006.09.0183 